\title{
Biodegradable Silicon-based Mesoporous Nanoparticles for Nanomedicine
}

\section{Baoyuan $\mathrm{Ma}^{1}$, Zhengfang Tian ${ }^{2}$ and Yufang Zhu ${ }^{12^{\star}}$}

${ }^{1}$ School of Materials Science and Engineering, University of Shanghai for Science and Technology, China

${ }^{2}$ Hubei Key Laboratory of Processing and Application of Catalytic Materials, College of Chemical Engineering, Huang gang Normal University, China

\section{Editorial}

Nanomaterials and nanotechnology have gained more and more attention due to their unique properties and intelligence in varieties of fields. In the field of medicine, many traditional problems have been solved with the development of drug delivery nanosystems. Compared with traditional drug administrations, the drug delivery nanosystems endow more accuracy of delivery and higher utilization rate of drugs, and thereby reduce the side effects and enhance the therapeutic efficiency.

For drug delivery nanosystems, the nanocarriers are vital for controlled drug release and imaging. To date, a variety of nanocarriers with different nanostructures have been designed and synthesized. Among them, mesoporous silica nanoparticles (MSNs) are considered to be effective nanocarriers due to their large surface area, high pore volume and controllable mesoporous structures, and they have been widely studied for drug delivery [1-3], diagnostic imaging [4], biosensing, synergistic cancer therapy [5,6], etc. For example, Yao et al. [7] designed an intelligent drug delivery nanosystem that graphene quantum dots (GQDs) were capped on the MSNs surface through the electrostatic interaction between GQDs and MSNs, which showed pHresponsive drug release behavior and good photothermal effect, and thus had great potential in chemo-photothermal synergistic cancer therapy. However, MSNs are faced with great challenges from basic research to clinical application. Due to the slow degradation rate of MSNs [8], their biocompatibility and biodegradability are poor. It has been well known that the condensation degree of Si-O-Si bond obviously affects the biodegradation rate of MSNs. To solve this problem, some strategies have been proposed to improve the biodegradability of MSNs, and the inorganic-organic hybridization and metal ion-doping are the most effective strategies.

The hybridization of organic functional groups in the MSNs frameworks induces the formation of mesoporous organosilica. Nowadays, various organic groups had been introduced into MSNs frameworks, such as $-\mathrm{CH}_{2}-\mathrm{CH}_{2}-,-\mathrm{CH}=\mathrm{CH}-,-\mathrm{S}-\mathrm{S}-$, etc. most of these organic functional groups are not biologically active, which limits the use in biomedical applications. However, disulfide bond (-S-S-) has good physiological activity and can be broken in the reducing environment. Generally, high level of glutathione (GSH) is in tumor tissue. Mesoporous organosilica nanoparticles (MONs) [9] hybridized with disulfide bond can degrade rapidly when MONs arrives in tumor tissue. Therefore, MONs hybridized with disulfide bond has good biodegradability, and this responsive degradability can trigger drug release, which is beneficial to be drug delivery nanocarriers. Recently, Huang et al. [10] proposed a simple and versatile method to synthesize hollow mesoporous organosilica nanoparticles (HMONs) with disulfide bond in the frameworks $(<50 \mathrm{~nm})$. All of HMONs have degraded into collapsed small pieces after immersing in SBF solution containing GSH $(10 \mathrm{mM})$ for 14 days, and only very few HMONs with collapsed nanostructures were observable after 7 days culture with breast cancer line $4 \mathrm{~T} 1$ cells, which indicated the easy biodegradation behavior of HMONs intracellularly. Furthermore, the DOX loading rate in HMONs is as high as $36.1 \%$, which is promising as a drug delivery nanocarrier. Using HMONs as biodegradable nanocarriers, Huang et al. [11] anchored the sonosensitizers (protoporphyrin, PpIX) into HMONs by an amidation reaction, and paramagnetic manganese $(\mathrm{Mn})$ ions were chelated into the porphyrin ring based on the metalloporphyrin chemistry. Thus, metalloporphyrin-encapsulated biodegradable nanosystems can achieve magnetic resonance imaging (MRI)-guided tumor sonodynamic therapy. Li et al. [12] prepared a biodegradable drug delivery nanosystem based on disulfide-hybridized HMONs functionalized with protoporphyrin and arginine-glycine-aspartic acid, which can target to hepatocellular carcinoma cells, and control drug release under ultrasound irradiation, enhancing the therapeutic efficiency. Wu et al. [13] constructed a biodegradable nanosystem that consisted of ultra-small $\mathrm{Cu}_{2-\mathrm{x}} \mathrm{Se}$ nanoparticles and disulfidehybridized HMONs, and this nanosystem showed multifunctionality with photoacoustic (PA) imaging, tumor-specific GSH-triggered biodegradation and on-demand releasing drugs for glioblastoma multiforme treatment in combination with focused ultrasound.

Organic-inorganic hybridization is an effective strategy to improve the biodegradation behavior of mesoporous silica nanoparticles. However, the organic-inorganic hybridization strategy suffers from the high-cost of the bissilylated organosilica precursors and the difficulty to control the synthesis process due to different hydrolysis/condensation rate of organosilica precursors. Nowadays, the doping of metal ions into the mesoporous silica frameworks is another effective strategy to improve the biodegradability of mesoporous silica nanoparticles. For example, Kristina et al. [14] doped ferric iron $\left(\mathrm{Fe}^{3+}\right)$ into the silica nanoshells by a sol-gel method, and these $\mathrm{Fe}^{3+}$-doped silica nanoshells could completely degrade within 20-25 days in normal physiological environment. Recently, Yu et al. [15] doped manganese (Mn) ions into the framework of mesoporous silica by Mn extraction strategy, and the incorporation of $\mathrm{Mn}-\mathrm{O}$ bond in the Si-O-Si frameworks significantly improved the biodegradability of MSNs, because the Mn-O bond could be rapidly degraded in a reducing or weak acid environment. On the other hand, the breaking of $\mathrm{Mn}-\mathrm{O}$ bond and releasing of $\mathrm{Mn}$ ions can obviously enhance the $\mathrm{T}_{1}$-weighted MRI performance for efficient tumor diagnosis imaging. Yu et al. [16] also directly doped magnesium $(\mathrm{Mg})$ into the framework of MSNs by generic dissolution and regrowth method to form magnesium silicate nanocarriers, and they found that magnesium silicate nanocarriers can respond to the mild acidic environment of tumor tissue, resulting in the rapid decomposition and

*Corresponding author:Yufang Zhu, School of Materials Science and Engineering, University of Shanghai for Science and Technology, 516 Jungong Road, Shanghai, 200093, China, Tel: +86-21-55271663; E-mail: zjf2412@163.com

Received November 12, 2018; Accepted November 19, 2018; Published November 26, 2018

Citation: Baoyuan Ma, Tian Z, Zhu Y (2018) Biodegradable Silicon-based Mesoporous Nanoparticles for Nanomedicine. J Bioanal Biomed 10:e159. doi:10.4172/1948-593X.1000e159

Copyright: @2018 Baoyuan Ma, et al. This is an open-access article distributed under the terms of the Creative Commons Attribution License, which permits unrestricted use, distribution, and reproduction in any medium, provided the original author and source are credited. 
Citation: Baoyuan Ma, Tian Z, Zhu Y (2018) Biodegradable Silicon-based Mesoporous Nanoparticles for Nanomedicine. J Bioanal Biomed 10:e159. doi:10.4172/1948-593X.1000e159

biodegradation of silica frameworks. Wang et al. [17] used a dissolutionregeneration strategy to synthesize biodegradable iron engineered MSNs frameworks (designated as $\mathrm{rFeOx}-\mathrm{HMSNs}$ ). On one hand, $\mathrm{rFeOx}$ HMSNs could rapidly degrade due to the mild acidic environment of cancer cells. On the other hand, $\mathrm{rFeOx}-\mathrm{HMSN}$ could kill cancer cells via the production of highly toxic hydroxyl radicals $(\cdot \mathrm{OH})$ owing to the Fenton reaction between the overexpressed hydrogen peroxide and $\mathrm{Fe}^{3+}$ in cancer cells. Furthermore, rFeOx-HMSNs can also be used as $\mathrm{T}_{2}$-weighted MRI contrast agents.

To date, organic-inorganic hybridization and metal ion-doping strategies have been widely used to synthesize biodegradable siliconbased mesoporous nanoparticles. These mesoporous nanoparticles are biocompatible and biodegradable; the degradation rate could be adjusted by changing the amount of disulfide bond or metal-O bond in the mesoporous silica frameworks. Using biodegradable silicon-based mesoporous nanoparticles as nanocarriers, the drug loading capacity is high, and the drug release could be responded to the microenvironment of cancer cells, enhancing the chemotherapeutic efficiency. On the other hand, these mesoporous nanoparticles can easily combine with other functional components, such as sonosensitizer, photosensitizer, targeting ligand, etc. for diagnosis and imaging, and thereby achieve the synergistic therapy and diagnosis. Therefore, biodegradable siliconbased mesoporous nanoparticles would be promising in nanomedicine in the future.

\section{Acknowledgement}

The authors gratefully acknowledge the support by the National Natural Science Foundation of China (51572172).

\section{References}

1. Zhu Y (2013) Mesoporous silica nanoparticles with a core-shell structure for drug delivery. J Bioanal Biomed 5: 3

2. Zhu Y, Ikoma T, Hanagata N, Kaskel S (2010) Rattle-type $\mathrm{Fe}_{3} \mathrm{O}_{4} @ \mathrm{SiO}_{2}$ hollow mesoporous spheres as carriers for drug delivery. Small 3: 471-478.

3. Zhu Y, Shi J, Shen W, Dong X, Feng J, et al. (2005) Stimuli-responsive controlled drug release from a hollow mesoporous silica sphere/polyelectrolyte multilayer core-shell structure. Angew Che 44: 5083-5087.

4. Tang F, Li L, Chen D (2012) Mesoporous silica nanoparticles: Synthesis, biocompatibility and drug delivery. Adv Mater 24: 1504-1534.
5. Chen Y, Chen H, Zeng D, Tian Y, Chen F, et al. (2010) Core/shell structured hollow mesoporous nanocapsules: a potential platform for simultaneous cell imaging and anticancer drug delivery. ACS Nano 4: 6001-6013.

6. Du X, Li X, Xiong L, Zhang X, Kleitz F, et al. (2016) Mesoporous silica nanoparticles with organo-bridged silsesquioxane framework as innovative platforms for bioimaging and therapeutic agent delivery. Biomaterials 91: 90127.

7. Yao X, Tian Z, Liu J, Zhu Y, Hanagata N (2017) Mesoporous silica nanoparticles capped with graphene quantum dots for potential chemo-photothermal synergistic cancer therapy. Langmuir 33: 591-599.

8. Peng YK, Tseng YJ, Liu CL, Chou SW, Chen YW, et al. (2015) One-step synthesis of degradable $\mathrm{T} 1-\mathrm{FeOOH}$ functionalized hollow mesoporous silica nanocomposites from mesoporous silica spheres. Nanoscale 7: 2676-2687.

9. Chen Y, Shi J (2016) Chemistry of mesoporous organosilica in nanotechnology: molecularly organic-inorganic hybridization into frameworks. Adv Mater 28 3235-3272.

10. Huang $P$, Chen $Y$, Lin $H, Y u ~ L$, Zhang $L$, et al. (2017) Molecularly organic/ inorganic hybrid hollow mesoporous organosilica nanocapsules with tumorspecific biodegradability and enhanced chemotherapeutic functionality Biomaterials 125: 23-37.

11. Huang P, Qian X, Chen Y, Yu L, Lin H, et al. (2017) Metalloporphyrinencapsulated biodegradable nanosystems for highly efficient magnetic resonance imaging-guided sonodynamic cancer therapy. J Am Chem Soc 139: $1275-1284$.

12. Li Z, Han J, Yu L, Qian X, Xing H, et al. (2018) Synergistic sonodynamic chemotherapeutic suppression of hepatocellular carcinoma by targeted biodegradable mesoporous nanosonosensitizers. Adv Funct Mater 28 1800145.

13. Wu M, Chen W, Chen Y, Zhang H, Liu C, et al. (2018) Focused ultrasoundaugmented delivery of biodegradable multifunctional nanoplatforms for imaging-guided brain tumor treatment. Adv Sci 5: 1700474

14. Pohaku Mitchell KK, Liberman A, Kummel AC, Trogler WC (2012) Iron(III)doped, silica nanoshells: a biodegradable form of silica. J Am Chem Soc 134 13997-14003.

15. Yu L, Chen Y, Wu M, Cai X, Yao H, et al. (2016) "Manganese extraction" strategy enables tumor-sensitive biodegradability and theranostics of nanoparticles. J Am Chem Soc 138: 9881-9894.

6. $\mathrm{Yu} \mathrm{L}$, Chen $\mathrm{Y}$, Lin $\mathrm{H}$, Gao $\mathrm{S}$, Chen $\mathrm{H}$, et al. (2018) Magnesium-engineered silica framework for ph-accelerated biodegradation and dnazyme-triggered chemotherapy. Small 14: 1800708.

17. Wang L, Huo M, Chen Y, Shi J (2018) Iron-engineered mesoporous silica nanocatalyst with biodegradable and catalytic framework for tumor-specific therapy. Biomaterials 163: 1-13. 